University of Wollongong

Research Online

Faculty of Arts, Social Sciences and Humanities

- Papers

Faculty of Arts, Social Sciences \& Humanities

$1-1-2020$

Development of Frontal EEG Differences Between Eyes-Closed and EyesOpen Resting Conditions in Children: Data From a Single-Channel DrySensor Portable Device

Stuart J. Johnstone

University of Wollongong, sjohnsto@uow.edu.au

Han Jiang

University of Wollongong, hjiang@uow.edu.au

Li Sun

Jeffrey Rogers

Joaquin Valderrama

See next page for additional authors

Follow this and additional works at: https://ro.uow.edu.au/asshpapers

Research Online is the open access institutional repository for the University of Wollongong. For further information contact the UOW Library: research-pubs@uow.edu.au 


\title{
Development of Frontal EEG Differences Between Eyes-Closed and Eyes-Open Resting Conditions in Children: Data From a Single-Channel Dry-Sensor Portable Device
}

\author{
Abstract \\ EEG and Clinical Neuroscience Society (ECNS) 2020. Changes in EEG when moving from an eyes-closed \\ to an eyes-open resting condition result from bottom-up sensory processing and have been referred to as \\ activation. In children, activation is characterized by a global reduction in alpha, frontally present \\ reductions for delta and theta, and a frontal increase for beta. The present study aimed to replicate frontal \\ EEG activation effects using single-channel, dry-sensor EEG, and to extend current understanding by \\ examining developmental change in children. Frontal EEG was recorded using a single-channel, dry- \\ sensor EEG device while 182 children aged 7 to 12 years completed eyes-closed resting (EC), eyes-open \\ resting (EO), and focus (FO) tasks. Results indicated that frontal delta, theta, and alpha power were \\ reduced, and frontal beta power was increased, in the EO compared with the EC condition. Exploratory \\ analysis of a form of top-down activation showed that frontal beta power was increased in the FO \\ compared with to the EO condition, with no differences for other bands. The activation effects were \\ robust at the individual level. The bottom-up activation effects reduced with age for frontal delta and \\ theta, increased for frontal alpha, with no developmental change for top-down or bottom-up frontal beta \\ activation. These findings contribute further to validation of the single-channel, dry-sensor, frontal EEG \\ and provide support for use in a range of medical, therapeutic, and clinical domains.
}

\section{Publication Details}

Johnstone, S., Jiang, H., Sun, L., Rogers, J., Valderrama, J. \& Zhang, D. (2020). Development of Frontal EEG Differences Between Eyes-Closed and Eyes-Open Resting Conditions in Children: Data From a SingleChannel Dry-Sensor Portable Device. Clinical EEG and Neuroscience,

\section{Authors}

Stuart J. Johnstone, Han Jiang, Li Sun, Jeffrey Rogers, Joaquin Valderrama, and Dawei Zhang 


\section{Title}

Development of frontal EEG differences between eyes-closed and eyes-open resting conditions in children: data from a single-channel dry-sensor portable device.

\section{Authors}

Stuart J Johnstone ${ }^{\mathrm{a}}$, Han Jiang ${ }^{\mathrm{b}}$, Li Sun ${ }^{\mathrm{c}, \mathrm{d}}$, Jeffrey M Rogers ${ }^{\mathrm{e}}$, Joaquin Valderrama ${ }^{\mathrm{f}, \mathrm{g}, \mathrm{h}}$, Dawei Zhang ${ }^{\mathrm{i}}$

\section{Affiliations}

${ }^{a}$ School of Psychology, Brain \& Behaviour Research Institute, University of Wollongong, Australia

${ }^{\mathrm{b}}$ School of Special Education, Zhejiang Normal University, Hangzhou, China

${ }^{c}$ Peking University Sixth Hospital and Institute of Mental Health, Beijing, China

${ }^{d}$ National Clinical Research Centre for Mental Disorders \& Key Laboratory of Mental Health, Ministry of Health (Peking University), Beijing, China

${ }^{\mathrm{e}}$ Faculty of Health Sciences, University of Sydney, Camperdown, Australia

${ }^{\mathrm{f}}$ National Acoustic Laboratories, Sydney, Australia

${ }^{g}$ Department of Linguistics, Macquarie University, Sydney, Australia

${ }^{\mathrm{h}}$ The HEARing CRC, Melbourne, Australia

${ }^{i}$ Department of Neuroscience, Karolinska Institute, Stockholm, Sweden

\section{Corresponding author}

Stuart J Johnstone, School of Psychology, University of Wollongong, Wollongong, NSW 2522 Australia. Emailsjohnsto@uow.edu.au 


\begin{abstract}
Changes in EEG when moving from an eyes-closed to an eyes-open resting condition result from bottom-up sensory processing and have been referred to as activation. In children, activation is characterised by a global reduction in alpha, frontally-present reductions for delta and theta, and a frontal increase for beta. The present study aimed to replicate frontal EEG activation effects using single-channel, dry-sensor EEG, and to extend current understanding by examining developmental change in children. Frontal EEG was recorded using a single-channel, dry-sensor EEG device while 182 children aged 7-12 years completed eyes-closed resting (EC), eyes-open resting (EO), and focus (FO) tasks. Results indicated that frontal delta, theta, and alpha power were reduced, and frontal beta power was increased, in the EO compared to the EC condition. Exploratory analysis of a form of top-down activation showed that frontal beta power was increased in FO compared to EO, with no differences for other bands. These activation effects were robust at the individual level. The bottom-up activation effects reduced with age for frontal delta and theta, increased for frontal alpha, with no developmental change for top-down or bottom-up frontal beta activation. These findings contribute further to validation of the single-channel, dry-sensor, frontal EEG and provide support for use in a range of medical, therapeutic, and clinical domains.
\end{abstract}

\title{
Keywords
}

EEG, Frontal, Development, Children, Activation, Arousal 


\section{Introduction}

The Cognitive Energetic Model (CEM) highlights the role of effort as well as tonic and phasic physiological activity in the efficient engagement of computational/cognitive processes and executive functions, with these factors determining the overall efficiency of information processing ${ }^{1}$. The tonic physiological aspect has been referred to as arousal ${ }^{a}$, defined as the current energetic level of the organism, and can be measured via skin conductance level (SCL) or brain electrical activity using electroencephalography (EEG). Increases in SCL, indicative of increased arousal, are associated with reductions in EEG power in the alpha frequency band across the scalp ${ }^{2}$. Alpha power is an inverse measure of arousal in children ${ }^{3}$ and adults ${ }^{4}$. The phasic physiological aspect of CEM has been referred to activation ${ }^{b}$, defined as a separable state measure reflecting the task-related mobilisation of energy (relative to a baseline level of arousal) needed for task performance ${ }^{2}$. Activation can be measured by examining the arousal difference between eyes-open resting and eyes-closed resting conditions, with the change in arousal level due to the increased sensory input in the eyes-open condition indicative of activation ${ }^{5}$. As would be predicted by the CEM theoretical framework, activation has been shown to contribute to task performance in adults 2,6-8 and executive functions (EFs) in children ${ }^{9}$. In children, the most prominent EEG indicator of activation is a global reduction in alpha power in the eyes-open condition, along with more regionally specific reductions in delta (focal frontal reduction, larger in the right hemisphere) and theta (larger reductions in frontal and posterior hemispheric regions) power, and a reduction in beta power in the posterior brain region and an increase in beta power in the frontal region ${ }^{10}$.

While EEG activation effects are similar in children ${ }^{10}$, young adults ${ }^{4}$, and older adults ${ }^{5}$, little is known about developmental change in arousal or activation during childhood. For the alpha band, it has been shown that frontal alpha power increases with age under eyes-closed conditions in this age range ${ }^{11}$. The current study will seek to confirm this effect and the inferred possible increase in alpha activation with age in this age range. An aim of the current study was to examine developmental change in each traditional EEG band in the 7-12 year age-range. Activation changes in other bands are also expected given literature indicating a decline in the absolute power of all bands, especially the slow waves ${ }^{11-13}$, along with evidence showing that functional information processing networks become more organized; both linked to structural changes in the brain such as synaptic pruning ${ }^{14}$.

EEG activation related to the opening of the eyes primarily results from increased visual system activity ${ }^{15-17}$. This type of activation is derived under resting (i.e. no task) conditions is a "bottom-up" process as it results from a simple passive change in sensory input. Another type of activation has also been considered by examining EEG changes from an eyes-open resting baseline to a cognitive task; termed taskrelated activation ${ }^{18}$. The current study sought to explore an extension of the activation concept to consider

\footnotetext{
${ }^{\text {a }}$ Originally termed activation in CEM, but referred to as arousal here in line with an empirically validated definition ${ }^{2}$ widely accepted in the literature.

${ }^{\mathrm{b}}$ Originally termed arousal in CEM, but referred to as activation here in line with an empirically validated definition ${ }^{2}$ widely accepted in the literature.
} 
"top-down" activation resulting from instructed modulation of a psychological state; in this case, attention, in accord with previous work ${ }^{19}$. This type of top-down activation, termed "attentional activation" here, aims to provide an index of EEG changes between an eyes-open resting state and an elevated attentional state that is less confounded by ongoing task-related cognitive processing, and thus could be considered a relatively pure EEG index of a top-down modulation of attention.

Previous investigations in this area have considered the topographic nature of EEG in eyes-open and eyes-closed resting conditions. In the current study, we aimed to determine if the established activation effects, all of which were either global or present in the frontal region, could be replicated in a single frontal EEG recording location using a dry-sensor, portable, recording device. Further, we aimed to explore how attentional activation was represented frontally. The single-channel, dry-sensor EEG has established concurrent validity via comparisons to a research system, and comparative validity via sensitivity to variations in psychological state ${ }^{20,21}$. The EEG is stable within recording sessions ${ }^{21}$ and reliable over short (one-day) and longer (one-week and one-month) retest-intervals ${ }^{22}$, in accord with lab-based EEG recording systems ${ }^{23,24}$ and $\mathrm{fMRI}{ }^{25}$. In addition to the limitation of only having one recording location ${ }^{20}$, other noted limitations relate to blink detection accuracy ${ }^{26}$, susceptibility to eye and muscle artifacts ${ }^{27}$, and estimation of spectral power at frequencies less than $4 \mathrm{~Hz}^{21,20}$. In terms of applications, frontal EEG from a single-channel portable device has been found to identify distinct electrophysiological profiles for stroke patients ${ }^{28}$, have prognostic value for post-stroke cognitive performance ${ }^{29,30}$, and reveal brain activity differences in children with foetal alcohol syndrome ${ }^{31}$. Further, this frontal EEG has been used in interventions for children with AD/HD ${ }^{32-35}$ and anxiety ${ }^{36,37}$, and found suitable for use in generation of auditory event-related potentials for braincomputer interface (BCI) applications ${ }^{38}$. Indeed, mobile EEG approaches are thought to have the potential to open unprecedented possibilities in the investigation of psychopathological mechanisms of neurodevelopmental disorders and the identification of EEG biomarkers ${ }^{39}$.

In summary, the present study aimed to replicate previously reported EEG activation effects in children using frontal, single-channel, dry-sensor EEG, extend current understanding of frontal arousal and activation by considering age effects in a large sample of children, and explore how top-down attentional activation is reflected in the frontal EEG of children. Specifically, it was predicted that the activation effects reported previously in children aged 8-12 years that were either global (e.g. reduction in alpha power) or present in the frontal region despite showing topographic variation (e.g. reductions in delta and theta power, and increase in beta power) would be replicated in the single-channel frontal EEG. Further, it was predicted that frontal EEG indices of arousal and activation would show developmental change in children. A reduction in frontal alpha power in the eyes-closed resting condition, not verified by SCL and thus considered a proxy measure, was used to index increased arousal. No specific predictions were made for attentional activation, rather frontal EEG in each band was examined by comparing an eyes-open resting condition and an instructed attention load condition, with age effects examined. 


\section{Materials and Methods}

\section{Participants}

One hundred and eighty two children aged between 7 and 12 years participated in this study, recruited from three primary schools in the Hangzhou region of China. Three age groups were formed with 70 children in the 7-8 year group ( 32 female, mean age 7.60 years), 70 children in the 9-10 year group ( 35 female, mean age 9.51 years), and 42 children in the 11-12 year group (21 female, mean age 11.74 years). Children were excluded from participation if they had a current or previous diagnosis of a psychological or psychiatric disorder, or had an average rating-per-item (ARI) score of 1 or more in DSM-V Inattention or Hyperactivity/Impulsivity symptom categories. Twenty two children from the original sample of 204 were excluded based on the ARI criteria, resulting in the sample described above. Zhejiang Normal University and the University of Wollongong and Illawarra Shoalhaven Local Health District Health and Medical Human Research Ethics Committee approved the research protocol prior to the commencement of data collection. The research was conducted in accordance with the approved guidelines, and each participant's parent(s) gave informed consent.

\section{Materials}

The Chinese version of the 40-item SNAP-IV rating scale was completed by each participant's parent ${ }^{40}$, examining participant's behaviour in terms of DSM-V items for AD/HD and ODD, items from the Conners Index Questionnaire ${ }^{41}$, and items from the IOWA Conners Rating Scale ${ }^{42}$, deriving six subscales. The parent form of the Chinese version of the SNAP-IV is both reliable and valid ${ }^{43}$, while the IOWA Conners Rating Scale has good internal consistency and test-retest reliability ${ }^{44}$. The items are rated on a 4-point scale from (0) 'not at all' to (3) 'very much'. ARI was calculated by averaging ratings for items in the DSM-V Inattention and DSM-V Hyperactivity/Impulsivity symptom categories separately, deriving scores that range from 0 to 3 .

\section{Procedure}

Each participant's parent(s) completed a demographic and screening questionnaire, and the SNAP-IV questionnaire. Separately, children were seated at a desk in front of a laptop computer, fitted with the EEG recording device, and then completed three EEG tasks with task order counterbalanced between participants. The eyes-open (EO) task required the child to look at a small image (simple smiling face) in the centre of the laptop screen for 2 minutes. The eyes-closed (EC) task required the child to sit with their eyes closed for 2 minutes. In the focus (FO) task children were instructed to "focus hard on the screen for 30 seconds" while viewing a simple coloured shape stimulus which slowly morphed in colour and shape over a $10 \mathrm{~s}$ period (e.g. red square morphing into yellow triangle, then morphing into blue square, etc.); there were two $30 \mathrm{~s}$ blocks. Participants were instructed to try to remain still during the EEG tasks and to direct their gaze to the stimuli on-screen to minimise muscle and eye movement artifacts. Each participant also completed three executive function tasks and several questionnaires that are not reported here. 


\section{EEG acquisition and quantification}

Continuous EEG was recorded at $512 \mathrm{~Hz}$ from scalp location FP1 referenced to the left ear using a portable EEG device (Mindwave Mobile, Neurosky, San Jose, California). The device consists of microchips and embedded firmware (ThinkGear, Neurosky, San Jose, California) and a 10 x $15 \mathrm{~mm}$ active electrode and ear-clip reference ground electrode contained within a light-weight comfortable headset. Raw EEG data was transmitted wirelessly by Bluetooth to the laptop computer for recording and subsequent off-line quantitative analysis.

EEG was analysed using Matlab functions from the 'Signal Processing' and 'Statistics and Machine Learning' toolboxes. The two $30 \mathrm{~s}$ blocks of the FO task were appended for analysis. Firstly, EEG from the $120 \mathrm{~s}$ EC task and the $120 \mathrm{~s}$ EO task were analysed, to allow comparison. Then, EEG from the $120 \mathrm{~s}$ EO task was analysed a second time, with analysis of a randomly chosen $60 \mathrm{~s}$ section of the $120 \mathrm{~s}$ trace, to allow direct comparison with the 60 s FO task.

Raw EEG data from the Mindwave device was band-pass filtered ( $4^{\text {th }}$ order Butterworth, $0.5-45 \mathrm{~Hz}$ ) with traces from the EO and FO tasks subject to an iterative template matching and suppression (IMTS) procedure to detect and suppress blink artifacts ${ }^{45}$. The ITMS approach consists of (1) an iterative process in which blink-artifacts are detected and the blink-artifact waveform is estimated, (2) generation of a signal modelling the blink-artifact, and (3) suppression of the blink-artifact model from the raw EEG. In this paper, the ITMS algorithm was implemented with 10 iterations, and initialized with the original blink-artifact template resampled at the sampling rate of the EEG device (i.e. $512 \mathrm{~Hz}$ ). The convergence of the algorithm to the blink-artifact morphology of each subject was validated by visual inspection. The EEG traces were then divided into 1 second epochs with a 50\% overlap, and windowed with a Hanning window. Epochs containing values exceeding $\pm 150 \mu \mathrm{V}$ were rejected. The remaining epochs were Fourier transformed and magnitude squared to obtain the power spectrum density from which the averaged spectral power $\left(\mu \mathrm{V}^{2}\right)$ was estimated in the four frequency bands: delta $(0.5$ to $3.5 \mathrm{~Hz}$ ), theta (3.5 to $7.5 \mathrm{~Hz}$ ), alpha (7.5 to $12.5 \mathrm{~Hz}$ ), and beta (12.5 to $25 \mathrm{~Hz}$ ). Visual inspection confirmed that the power spectrum density calculated in each subject and condition was free of abnormal peaks ${ }^{46}$.

\section{Data analysis}

Statistical analysis was carried out using IBM SPSS Statistics version 25, testing a general linear model. Assumptions of normality and sphericity were met for all EEG variables. For EEG epochs, a repeated measures ANOVA was conducted with Condition (EC, $120 \mathrm{~s} \mathrm{EO)} \mathrm{as} \mathrm{a} \mathrm{within-subjects} \mathrm{factor} \mathrm{and} \mathrm{Age} \mathrm{(7-8,} \mathrm{9-}$ $10,11-12$ years) as a between-subjects factor, with a similar analysis conducted comparing epochs in the $60 \mathrm{~s}$ EO quantification and FO. For arousal, absolute power in the alpha band in the EC condition was subject to a univariate ANOVA with Age (7-8, 9-10, 11-12 years) as a between-subjects factor, with planned contrasts allowing consideration of developmental change by comparing the 7-8 year group to both the 9-10 year and 11-12 year groups. For activation, separate repeated measures ANOVAs were conducted for the absolute 
power in each frequency band with Condition (EC, EO) as a within-subjects factor and Age (7-8, 9-10, 11-12 years) as a between-subjects factor, with simple effects within each age group separately examined post-hoc. A similar analysis was conducted for attentional activation, with Condition (EO, FO) as the within-subjects factor. Effect sizes for significant Age group comparisons are reported using Cohen's $d$ for groups with equal sample sizes, and Hedges' $g$ for groups with unequal sample sizes. Pearson correlations of arousal and activation were computed for alpha power separately within each age group, to confirm the arousal-activation relationship in frontal EEG.

\section{Results}

EEG epochs

There was no significant difference between the number of epochs in the EC $(M=80.24, S D=27.78$, $95 \% \mathrm{CI}[73.84,86.63])$ and EO $(M=82.08, S D=28.14,95 \%$ CI $[75.72,88.43])$ conditions, or the 60 s EO $(M$ $=41.47, S D=13.95,95 \% \mathrm{CI}[37.89,45.67])$ and FO $(M=39.79, S D=14.78,95 \% \mathrm{CI}[36.41,43.17])$ conditions. In both comparisons, the Condition by Age interaction was not significant.

\section{Frontal EC alpha}

Developmental effects were examined for frontal alpha power in the EC condition. An Age main effect, $\mathrm{F}=4.691, \mathrm{p}=.010, \eta_{p}^{2}=0.050$, and planned contrasts indicated increased power ( $\mathrm{p}=.003$, Cohen's $d$ $=0.51)$ in the 9-10 year group $\left(M=32.65 \mu \mathrm{V}^{2}, S D=15.33,95 \%\right.$ CI $\left.[29.59,35.72]\right)$ compared to the 7-8 year group $\left(M=25.95 \mu \mathrm{V}^{2}, S D=10.53,95 \%\right.$ CI $\left.[22.86,29.04]\right)$ and no significant difference between the 11-12 year group $\left(M=30.26 \mu \mathrm{V}^{2}, S D=12.47,95 \% \mathrm{CI}[26.30,34.23]\right)$ and 7-8 year group.

\section{Frontal EC versus EO effects}

For activation, condition and developmental effects were examined for each band separately. Significance values for the main effects and interactions are shown in Table 1.

Frontal delta power showed a near-significant Age main effect, with planned contrasts indicating reduced power $(\mathrm{p}=.017$, Hedges' $g=0.42)$ in the $11-12$ year group $\left(M=51.42 \mu \mathrm{V}^{2}, S D=38.37,95 \% \mathrm{CI}\right.$ $[34.89,67.98])$ compared to the 7-8 year group $\left(M=76.95 \mu \mathrm{V}^{2}, S D=69.99,95 \%\right.$ CI $\left.[64.03,89.88]\right)$ and no difference between the 9-10 year group $\left(M=63.86 \mu \mathrm{V}^{2}, S D=54.36,95 \% \mathrm{CI}[51.03,76.69]\right)$ and the 7-8 year group. A Condition main effect indicated reduced power in the $\mathrm{EO}\left(M=49.87 \mu \mathrm{V}^{2}, S D=43.66,95 \% \mathrm{CI}\right.$ $[43.06,56.68])$ compared to the $\mathrm{EC}\left(M=78.29 \mu \mathrm{V}^{2}, S D=64.81,95 \%\right.$ CI $\left.[67.57,89.00]\right)$ condition. As shown in Figure 1, the Condition by Age interaction and simple effects revealed that the largest reduction was in the 7-8 year group $\left(M_{\text {diff }}=41.69 \mu \mathrm{V}^{2}, S D=39.24\right), \mathrm{F}=29.247, \mathrm{p}<.001, \eta_{p}^{2}=0.301$, followed by the $9-10$ year group $\left(M_{\text {diff }}=28.67 \mu \mathrm{V}^{2}, S D=19.74\right), \mathrm{F}=46.136, \mathrm{p}<.001, \eta_{p}^{2}=0.401$, and then the 11-12 year group $\left(M_{\text {diff }}=14.88 \mu \mathrm{V}^{2}, S D=4.48\right), \mathrm{F}=7.573, \mathrm{p}=.009, \eta_{p}^{2}=0.156$. 
Frontal theta power showed an Age main effect, with planned contrasts indicating reduced power $(\mathrm{p}<$ .000 , Hedges' $g=0.62)$ in the 11-12 year group $\left(M=32.76 \mu \mathrm{V}^{2}, S D=16.83,95 \%\right.$ CI $\left.[25.55,39.97]\right)$ compared to the 7-8 year group $\left(M=49.39 \mu \mathrm{V}^{2}, S D=31.43,95 \% \mathrm{CI}[43.76,55.02]\right)$ and no difference between the 9-10 year group $\left(M=43.11 \mu \mathrm{V}^{2}, S D=25.75,95 \% \mathrm{CI}[37.53,48.70]\right)$ and the 7-8 year group. A Condition main effect indicated reduced power in the EO $\left(M=35.44 \mu \mathrm{V}^{2}, S D=21.73,95 \% \mathrm{CI}[31.96\right.$, 38.95]) compared to the $\mathrm{EC}\left(M=48.07 \mu \mathrm{V}^{2}, S D=27.42,95 \% \mathrm{CI}[43.63,52.51]\right)$ condition. As shown in Figure 1, a Condition by Age interaction and simple effects indicated that the largest reduction was in the 7-8 year group $\left(M_{\text {diff }}=18.40 \mu \mathrm{V}^{2}, S D=8.05\right), \mathrm{F}=23.772, \mathrm{p}<.001, \eta_{p}^{2}=0.259$, followed by the 9-10 year group $\left(M_{\text {diff }}=12.83 \mu \mathrm{V}^{2}, S D=5.16\right), \mathrm{F}=36.715, \mathrm{p}<.001, \eta_{p}^{2}=0.347$, and then the $11-12$ year group $\left(M_{\text {diff }}=6.58\right.$ $\left.\mu \mathrm{V}^{2}, S D=3.87\right), \mathrm{F}=5.825, \mathrm{p}=.020, \eta_{p}^{2}=0.124$.

Frontal alpha power showed an Age main effect, with planned contrasts indicating that the 9-10 year group $\left(M=27.53 \mu \mathrm{V}^{2}, S D=13.36,95 \% \mathrm{CI}[25.22,29.84]\right)$ was increased ( $\mathrm{p}=.010$, Cohen's $\left.d=0.38\right)$ compared to the 7-8 year group $\left(M=23.18 \mu \mathrm{V}^{2}, S D=9.37,95 \% \mathrm{CI}[20.86,25.51]\right)$ and no difference between the 7-8 year group and 11-12 year group $\left(M=24.14 \mu \mathrm{V}^{2}, S D=9.18,95 \% \mathrm{CI}[21.16,27.12]\right)$. A Condition main effect indicated reduced power in the $\mathrm{EO}\left(M=20.28 \mu \mathrm{V}^{2}, S D=8.50,95 \% \mathrm{CI}[18.89,21.66]\right)$ compared to EC $\left(M=29.28 \mu \mathrm{V}^{2}, S D=12.78,95 \% \mathrm{CI}[27.66,31.59]\right)$ condition. As shown in Figure 1 , a Condition by Age interaction with simple effects indicated that the largest reduction was in the 11-12 year group $\left(M_{\text {diff }}=12.25 \mu \mathrm{V}^{2}, S D=6.59\right), \mathrm{F}=46.936, \mathrm{p}<.001, \eta_{p}^{2}=0.534$, followed by the 9-10 year group $\left(M_{\text {diff }}\right.$ $\left.=10.25 \mu \mathrm{V}^{2}, S D=3.95\right), \mathrm{F}=54.554, \mathrm{p}<.001, \eta_{p}^{2}=0.442$, and then the 7-8 year group $\left(M_{\text {diff }}=5.54 \mu \mathrm{V}^{2}, S D\right.$ $=2.31), \mathrm{F}=20.165, \mathrm{p}<.001, \eta_{p}^{2}=0.229$.

\section{-- INSERT TABLE 1 HERE -- -- INSERT FIGURE 1 HERE --}

Frontal beta power showed an Age main effect, with planned contrasts indicating that the 7-8 year group $\left(M=9.22 \mu \mathrm{V}^{2}, S D=6.00,95 \% \mathrm{CI}[8.07,10.37]\right)$ did not differ from the 9-10 year group $(M=10.11$ $\mu \mathrm{V}^{2}, S D=6.01,95 \%$ CI $\left.[8.97,11.25]\right)$ or the $11-12$ year $\left(M=7.65 \mu \mathrm{V}^{2}, S D=3.55,95 \% \mathrm{CI}[6.18,9.12]\right)$ group. A follow-up difference contrast confirmed a significant $(\mathrm{p}=.019$, Hedges' $g=0.38)$ reduction in beta power in the 11-12 year group compared to the mean of the 7-8 and 9-10 year groups. As shown in Figure 2, a Condition main effect indicated increased power in the EO $\left(M=10.98 \mu \mathrm{V}^{2}, S D=7.52,95 \%\right.$ CI $[9.75$, 12.22]) condition compared to the $\mathrm{EC}\left(M=7.00 \mu \mathrm{V}^{2}, S D=2.85,95 \% \mathrm{CI}[6.56,7.44]\right)$ condition. The Condition by Age interaction was not significant. 
For attentional activation, condition and developmental effects were examined for each band separately. For both frontal delta and theta power there were no significant Age or Condition main effects or Age by Condition interactions.

Frontal alpha power showed a borderline significant Age main effect, with planned contrasts indicating that the 7-8 year group $\left(M=21.01 \mu \mathrm{V}^{2}, S D=15.37,95 \% \mathrm{CI}[18.38,23.64]\right)$ did not differ from the 9-10 year group $\left(M=22.45 \mu \mathrm{V}^{2}, S D=12.23,95 \% \mathrm{CI}[19.83,25.08]\right)$ or the $11-12$ year $\left(M=17.14 \mu \mathrm{V}^{2}, S D\right.$ $=7.27,95 \% \mathrm{CI}[13.74,20.53])$ group. A follow-up difference contrast $(\mathrm{p}=.020$, Hedges' $g=0.41)$ confirmed reduced alpha power in the 11-12 year group compared to the mean of the 7-8 and 9-10 year groups. The Condition main effect and Condition by Age interaction were not significant.

Frontal beta power did not show an Age main effect. A significant Condition main effect indicated increased power in the $\mathrm{FO}\left(M=12.76 \mu \mathrm{V}^{2}, S D=11.81,95 \% \mathrm{CI}[10.88,14.65]\right)$ compared to the $\mathrm{EO}(M=$ $10.98 \mu \mathrm{V}^{2}, S D=7.50,95 \%$ CI $\left.[9.74,12.21]\right)$ condition - see Figure 2. The Condition by Age interaction was not significant.

\section{Correlations between frontal EC alpha and alpha activation}

The correlation between frontal alpha power frontal in the EC condition and alpha activation was significant in the 7-8 year group, $r=-.604, \mathrm{p}<.01, \mathrm{R}^{2}=.364$, the 9-10 year group, $r=-.675, \mathrm{p}<.01, \mathrm{R}^{2}=$ .455 , and the 11-12 year group, $r=-.883, \mathrm{p}<.01, \mathrm{R}^{2}=.780$.

\section{Follow-up analyses}

Additional analyses were conducted to consider Condition effects at the individual level. For activation, a difference score was calculated by subtracting EEG power in the EC from EO condition for those bands that showed significant Condition effects (i.e. each band). Similarly, for attentional activation a difference score was calculated by subtracting EEG power in the EO from FO condition for the beta band. Table 2 shows the percentage of the overall sample that showed reduced power in the delta, theta, and alpha bands, and increased power in the beta band for the EO-EC values, and showed increased power in the beta band for the FO-EO values. Figure 3 shows histograms of the difference scores.

\section{-- INSERT TABLE 2 HERE --$$
\text { -- INSERT FIGURE } 3 \text { HERE -- }
$$

\section{Discussion}

The current study aimed to replicate previously reported EEG activation effects in children using frontal, single-channel, dry-sensor EEG, extend current understanding of frontal arousal and activation by considering age effects in a large sample of children, and explore how top-down attentional activation is reflected in the frontal EEG of children. The sample of children had no current or previous diagnoses of any 
psychological or psychiatric disorder and showed very low levels of inattentive and hyperactive/impulsive behaviour, and hence can be considered typically-developing.

The activation data were obtained from a frontal scalp location using a single-channel, dry-sensor EEG recording device and replicate previous frontal activation effects in this age-range for delta, theta, alpha, and beta power. This includes eyes-closed to open reductions in alpha power that were global, reductions in delta and theta power reported to vary topographically and be present frontally in children ${ }^{10}$, young adults ${ }^{4}$, and older adults ${ }^{5}$. The eyes-closed to open increase in beta power in the frontal region has also been reported in children ${ }^{10}$ and young adults ${ }^{4}$, but not older adults ${ }^{5}$.

In line with previous EC data ${ }^{11}$, the frontal proxy index of arousal did show an effect of age, but the trend was not linear. Given the inverse relationship between alpha power and arousal ${ }^{4}$, this effect indicates that the youngest and oldest children showed higher resting arousal than the 9-10 year old children as measured with frontal EEG. Consideration of the pattern of means shows a large increase (decrease in resting arousal) in frontal alpha power from 7-8 to 9-10 years and only a minor reduction power from 9-10 to 11-12 years, may suggest a plateau in development over the age-range of the two older groups, although this cannot be confirmed without extending the sample to consider adolescents. Our index of arousal is different to the traditional approach and is thus referred to as a proxy index, as we have utilised only the frontal aspect of the previously established across-scalp global reduction in alpha power in eyes-open compared to eyes-closed conditions and not corroborated or correlated this effect with SCL. Despite this limitation, our protocol facilitated a large sample and provides insight into frontal developmental change in the child age-range.

Our results indicate that frontal EEG indices of activation show substantial developmental change in children. Frontal delta and theta activation effects both reduced as age increased. Thus, frontal EEG power in the lower frequency bands becomes more similar in these two resting conditions as age increases in children. While developmental change appears to be occurring in these EEG bands for both the EC and EO conditions, Figure 1 indicates that the change is larger in the EC condition - thus, the developmental effects may be driven more by changes in neural activity during a resting state with no visual information processing than during basic visual processing. Frontal alpha activation increased as age increased. Thus, frontal EEG power in the alpha band becomes more different in these two resting conditions as age increases in children. These findings confirm substantial developmental change in the frontal aspect of the alpha activation, an effect inferred previously ${ }^{11}$. Note that similar activation effects derived from 19-channels of EEG and examined topographically were reported to be unrelated to age in a small sample of children 8 to 12 years old with a mean age of 10.6 years ${ }^{10}$. Thus, the current study has added to our current understanding of developmental change in these indices of activation, with a frontal focus. It would be informative to consider the effect of age from a multi-channel topographic perspective in future research.

In line with the previously-reported multi-channel effects ${ }^{10}$, frontal beta activation did not change as a function of age. An increase in frontal beta power upon opening the eyes characterised bottom-up activation consistently across the 7 to 12 year age-range. This finding appears consistent with functional understanding 
of frontal activity in this frequency range, which is associated with cognitive engagement and stimulus assessment $^{47,48}$.

Attentional activation was seen in an increase in frontal beta power, with no significant differences between the EO and FO conditions for the other EEG bands frontally. The frontal beta activation effect was consistent across the 7 to 12 year age-range. Using the current methodology, which aimed to elicit top-down attention modulation independently of task-related cognitive/response demands, it is not possible to determine the extent to which children were able to modulate their attention level as instructed in the focus task, although the change in the beta band previously linked functionally to stimulus assessment may indicate they were, to a certain degree, able to complete the task as requested. The frontal beta effects for activation and attentional activation were similarly represented by increased power and no developmental change. In this way, an increase in frontal beta power appears to be common to both bottom-up and top-down activation ${ }^{19}$.

For each age group, children with lower frontal alpha power in the EC condition showed larger frontal alpha activation; the correlation increased with increasing age. Global alpha activation/reactivity has been interpreted as evidence that the alpha change is mainly due to an arousal increase, due to changes that support visual processing ${ }^{5}$. Our results suggest that this effect is measurable using a single frontal channel, and consistent in children. Future research could incorporate SCL as the "gold standard" measure of arousal to more accurately quantify and corroborate the frontal EEG indices of arousal. Decreased localised delta and theta in the EO condition has been seen as indicative of increased activation associated with unstructured visual processing of the immediate environment in the absence of a task ${ }^{5}$. Though localised, these effects were present frontally in previous studies ${ }^{4,5,10}$, and were measurable here using a single frontal channel with evidence of developmental change in children.

While the frontal alpha results reported here have been considered in an arousal/activation context, alpha activity has also been explained as cortical inhibition ${ }^{49-51}$. In this context, an alpha increase indicates that the measured region is more inhibited, whereas an alpha decrease indicates that the measured region is released from inhibition ${ }^{50}$. The inhibition explanation is based on observations that alpha activity is increased in task-irrelevant cortical regions ${ }^{52-54}$. From this perspective, the frontal alpha activation effect can be explained in terms of a release from cortical inhibition in this region, moving into a more active mode with visual stimulus input. Correspondingly, the developmental pattern reported here for the frontal alpha activation effect suggests that the extent to which the frontal region is less inhibited (more engaged) upon opening the eyes increases with age.

The current replication of previous activation effects derived from multi-channel, research-grade EEG systems in laboratory environments provides further evidence for the validity of frontal, single-channel, drysensor EEG. The EC and EO absolute band power values reported here were broadly similar to those previously reported in the frontal region in 8-12 year old children ${ }^{10}$, and frontal EC absolute power band values in children ${ }^{12}$. The activation effects showed reasonable consistency at the individual level, with the lowest prevalence for beta at $76 \%$ and the highest for alpha at $85 \%$. Prevalence of attentional activation was lower, at $58 \%$ for beta. This level of consistency is similar to $85 \%$ required for clinical application ${ }^{55}$ for alpha 
activation only, so caution should be utilised for use at the individual level at this stage. It may be possible to improve consistency. While likely influenced by electrophysiological individual differences, this consistency is also dependent on the extent to which children are able to understand the EEG task instructions and carry out the tasks as instructed. As the prevalence rates differed between age groups, developing age-specific instructions may help children more fully understand the task and complete it as instructed. As it was observed that younger children sometimes experienced difficulty staying on-task for the full 2 minutes of the EO and EC tasks, dividing the tasks into two 1-minute blocks may also be beneficial.

Our data processing approach attempted to address limitations that have been raised about accounting for artifacts when recording from one electrode ${ }^{26,27}$. We used the ITMS method ${ }^{45}$ to estimate and suppress the artifact derived from eye-blinks, however, it is plausible that the EEG is affected by some degree of electromyogram (EMG) activity such as eye movements, as well as frontalis and temporalis muscle contractions. Strong EMG activity can cause an overestimation of power at the higher-frequency bands, i.e. beta, and to some extent, alpha ${ }^{56}$; thus adding extra variability to the measures. Due to the lack of a source signal of EMG activity ${ }^{57}$, attenuating EMG artifact in single-channel EEG applications is challenging. Gasser et al. ${ }^{56}$ proposed a method in which the power in a higher-frequency band (e.g. 51-69 Hz) was used as an indirect estimate of EMG activity, and used this parameter as a predictor in a regression analysis. The authors of that study demonstrated that the proposed method efficiently corrected the artifact derived from EMG activity in subjects with pre-senile and senile Alzheimer Disease. Taking these results into account, we recommend the use of the EMG correction method proposed by Gasser et al. ${ }^{56}$ when evaluating subjects with any psychological or psychiatric disorder.

The limitations of recording EEG from only one frontal channel must be acknowledged. As well as presenting challenges for controlling for inevitable blink/eye movement related artifacts, single-channel data does not allow concurrent consideration of data from other brain regions. While these limitations would be insurmountable in certain contexts (e.g. for neurological examination), if the data can be shown to be valid and reliable and replicate known effects derived frontally from research grade EEG systems (as is the case in the present study), they may represent an acceptable trade-off and facilitate EEG recording for certain purposes outside of the laboratory context. For example, in addition to current use in hospital and therapeutic settings, frontal EEG recorded in this manner could provide an additional neurophysiological perspective in the clinical psychological domain. Given the value of resting-state EEG as a biomarker in psychological disorders ${ }^{58}$ and its sensitivity to developmental change ${ }^{14}$, the ability to record frontal EEG in a clinic setting in a convenient, technically simple, and low-cost manner could be very valuable. This EEG could inform the diagnostic process or provide an objective physiological index of treatment suitability, progression, or outcome. To realise such clinical potential the characteristics of the frontal EEG must be established through systematic research (as represented here), alongside the development of large-scale normative and clinical databases for comparisons of individual EEG profiles. The value of such an approach that utilises mobile EEG, thus facilitating large-scale EEG data collection, has been recently recognised ${ }^{39,59}$. 
These results presented here should be considered in light of several limitations. While the sample of children did not have any current or previous diagnoses of any psychological or psychiatric disorder and showed very low levels of inattentive and hyperactive/impulsive behaviour, we did not assess their basic cognitive functioning (e.g. as indicated by IQ) or learning ability. Although the ANOVA is robust to different participant numbers in between-group comparisons ${ }^{60}$, the smaller sample in the 11-12 year group may have affected the results. As mentioned above, we did not record SCL to confirm its relationship with eyes-closed resting alpha as a measure of arousal. The focus task was designed to be relatively free of task-related requirements and cognitive processing, and used a slowly morphing stimulus based on our pilot studies which showed that children reported that modulating attention in the absence of a stimulus or with just a simple shape stimulus (e.g. a blue square) difficult. While the children had no instructions related to the morphing shape stimulus, and were instructed only to "focus hard on the screen" putting emphasis on top-down attention modulation, the attentional activation effect may be at least partly due to the visual processing element.

\section{Conclusions}

The current study has replicated the frontal findings of previously reported EEG activation effects in children aged 7 to 12 years using frontal, single-channel, dry-sensor EEG. These findings contribute further to validation of the single-channel, dry-sensor, frontal EEG. Further, this study has extended current understanding of EEG activation effects to reveal developmental change for frontal delta, theta, and alpha activation and stability frontal beta activation across the 7 to 12 year age-range. We reported non-linear developmental change in a frontal proxy index of arousal. The exploration of top-down attentional activation revealed that it was characterised by an increase in frontal beta power only, with stability across the 7 to 12 year age-range. Future research could investigate the consistency of these findings in children from a western culture. Further, in line with the framework of the CEM, future studies might consider the relationship of arousal, activation, and attentional activation to cognitive/executive function performance, and consider these effects in children with neurodevelopmental disorders such as ASD or AD/HD.

\section{Funding}

This research received no specific grant from any funding agency in the public, commercial, or notfor-profit sectors.

\section{Declaration of conflicting interests}

The Authors declare that there is no conflict of interest. 


\section{References}

1. Sanders A. Towards a model of stress and performance. Acta Psychol (Amst). 1983;(53):61-97.

2. Barry RJ, Clarke AR, McCarthy R, Selikowitz M, Rushby JA. Arousal and activation in a continuous performance task: An exploration of state effects in normal children. J Psychophysiol. 2005;19(2):9199. doi:10.1027/0269-8803.19.2.91

3. Barry RJ, Clarke AR, McCarthy R, Selikowitz M, Rushby JA, Ploskova E. EEG differences in children as a function of resting-state arousal level. Clin Neurophysiol. 2004;115(2):402-408. doi:10.1016/S1388-2457(03)00343-2

4. Barry RJ, Clarke AR, Johnstone SJ, Magee CA, Rushby JA. EEG differences between eyes-closed and eyes-open resting conditions. Clin Neurophysiol. 2007;118(12):2765-2773. doi:10.1016/j.clinph.2007.07.028

5. Barry RJ, De Blasio FM. EEG differences between eyes-closed and eyes-open resting remain in healthy ageing. Biol Psychol. 2017;129(August):293-304. doi:10.1016/j.biopsycho.2017.09.010

6. VaezMousavi SM, Barry RJ, Rushby JA, Clarke AR. Arousal and activation effects on physiological and behavioral responding during a continuous performance task. Acta Neurobiol Exp (Wars). 2007;67(4):461-470.

7. Karamacoska D, Barry RJ, Steiner GZ. Resting state intrinsic EEG impacts on go stimulus-response processes. Psychophysiology. 2017;54(6):894-903. doi:10.1111/psyp.12851

8. Tenke CE, Kayser J, Abraham K, Alvarenga JE, Bruder GE. Posterior EEG alpha at rest and during task performance: Comparison of current source density and field potential measures. Int $J$ Psychophysiol. 2015;97(3):299-309. doi:10.1016/j.ijpsycho.2015.05.011

9. Choon Guan Lim B, Lee T-S, Guan C, et al. Effectiveness of a Brain-Computer Interface Based Programme for the Treatment of ADHD: A Pilot Study. Psychopharmacol Bull. 2010;4343(731):7382.

10. Barry RJ, Clarke AR, Johnstone SJ, Brown CR. EEG differences in children between eyes-closed and eyes-open resting conditions. Clin Neurophysiol. 2009;120(10):1806-1811. doi:10.1016/j.clinph.2009.08.006

11. Miskovic V, Ma X, Chou CA, et al. Developmental changes in spontaneous electrocortical activity and network organization from early to late childhood. Neuroimage. 2015;118:237-247. doi:10.1016/j.neuroimage.2015.06.013

12. Clarke AR, Barry RJ, McCarthy R, Selikowitz M. Age and sex effects in the EEG: development of the normal child. Clin Neurophysiol. 2001;112:815-826. 
13. Segalowitz SJ, Santesso DL, Jetha MK. Electrophysiological changes during adolescence: A review. Brain Cogn. 2010;72(1):86-100. doi:10.1016/j.bandc.2009.10.003

14. Anderson AJ, Perone S. Developmental change in the resting state electroencephalogram: Insights into cognition and the brain. Brain Cogn. 2018;126(July):40-52. doi:10.1016/j.bandc.2018.08.001

15. Volavka J, Matoušek M, Roubíček J. Mental arithmetic and eye opening. An EEG frequency analysis and GSR study. Electroencephalogr Clin Neurophysiol. 1967;22(2):174-176. doi:10.1016/00134694(67)90158-7

16. Härdle W, Gasser T, Bächer P. EEG-responsiveness to eye opening and closing in mildly retarded children compared to a control group. Biol Psychol. 1984;18(3):185-199. doi:10.1016/03010511(84)90002-4

17. Gale A, Coles M, Boyd E. Variation in visual input and the occipital EEG: II. Psychon Sci. 1971;23(1):99-100. doi:10.3758/BF03336026

18. Karamacoska D, Barry RJ, Steiner GZ, Coleman EP, Wilson EJ. Intrinsic EEG and task-related changes in EEG affect Go/NoGo task performance. Int J Psychophysiol. 2018;125(December 2017):17-28. doi:10.1016/j.ijpsycho.2018.01.015

19. Buschman TJ, Miller EK. Top-Down Versus Bottom-Up Control of Attention in the Prefrontal and. Science (80- ). 2007;315(March):1860-1863.

20. Johnstone SJ, Blackman R, Bruggemann JM. EEG from a single-channel dry-sensor recording device. Clin EEG Neurosci. 2012;43(2). doi:10.1177/1550059411435857

21. Rieiro H, Diaz-Piedra C, Morales JM, et al. Validation of Electroencephalographic Recordings Obtained with a Consumer-Grade, Single Dry Electrode, Low-Cost Device: A Comparative Study. Sensors (Basel). 2019;19(12):1-18. doi:10.3390/s19122808

22. Rogers JM, Johnstone SJ, Aminov A, Donnelly J, Wilson PH. Test-retest reliability of a singlechannel, wireless EEG system. Int J Psychophysiol. 2016;106. doi:10.1016/j.ijpsycho.2016.06.006

23. Corsi-Cabrera M, Galindo-Vilchis L, del-Río-Portilla Y, Arce C, Ramos-Loyo J. Within-subject reliability and inter-session stability of EEG power and coherent activity in women evaluated monthly over nine months. Clin Neurophysiol. 2007;118(1):9-21. doi:10.1016/j.clinph.2006.08.013

24. Cannon RL, Baldwin DR, Shaw TL, et al. Reliability of quantitative EEG (qEEG) measures and LORETA current source density at 30 days. Neurosci Lett. 2012;518(1):27-31. doi:10.1016/j.neulet.2012.04.035

25. Zuo XN, Xing XX. Test-retest reliabilities of resting-state FMRI measurements in human brain functional connectomics: A systems neuroscience perspective. Neurosci Biobehav Rev. 2014;45:100118. doi:10.1016/j.neubiorev.2014.05.009 
26. Maskeliunas R, Damasevicius R, Martisius I, Vasiljevas M. Consumer grade EEG devices: are they usable for control tasks? PeerJ. 2016;4:e1746. doi:10.7717/peerj.1746

27. Ratti E, Waninger S, Berka C, Ruffini G, Verma A. Comparison of Medical and Consumer Wireless EEG Systems for Use in Clinical Trials. Front Hum Neurosci. 2017;11(August):1-7. doi:10.3389/fnhum.2017.00398

28. Rogers JM, Bechara J, Middleton S, Johnstone SJ. Acute EEG Patterns Associated With Transient Ischemic Attack. Clin EEG Neurosci. 2018. doi:10.1177/1550059418790708

29. Animov A, Rogers JM, Johnstone SJ, Middleton S, Wilson PH. Acute single channel EEG predictors of cognitive function after stroke. Int J Stroke. 2017;12(2):18. doi:10.1177/1747493017714154

30. Rogers J, Middleton S, Wilson PH, et al. Predicting functional outcomes after stroke: an observational study of acute single-channel EEG. Top Stroke Rehabil. 2019;00(00):1-12.

doi:10.1080/10749357.2019.1673576

31. Hemington KS, Reynolds JN. Electroencephalographic correlates of working memory deficits in children with Fetal Alcohol Spectrum Disorder using a single-electrode pair recording device. Clin Neurophysiol. 2014;125(12):2364-2371. doi:10.1016/j.clinph.2014.03.025

32. Johnstone SJ, Roodenrys S, Blackman R, et al. Neurocognitive training for children with and without AD/HD. ADHD Atten Deficit Hyperact Disord. 2012;4(1). doi:10.1007/s12402-011-0069-8

33. Jiang H, Johnstone SJ. A Preliminary Multiple Case Report of Neurocognitive Training for Children With AD/HD in China. SAGE Open. 2015;5(2):2158244015586811-. doi:10.1177/2158244015586811

34. Johnstone SJ, Roodenrys SJ, Johnson K, Bonfield R, Bennett SJ. Game-based combined cognitive and neurofeedback training using Focus Pocus reduces symptom severity in children with diagnosed AD/HD and subclinical AD/HD. Int J Psychophysiol. 2017;116. doi:10.1016/j.ijpsycho.2017.02.015

35. Jiang H, Johnstone SJ, Sun L, Zhang D-W. Effect of Neurocognitive Training for Children With ADHD at Improving Academic Engagement in Two Learning Settings. J Atten Disord. 2018:108705471879993. doi:10.1177/1087054718799931

36. Wijnhoven LAMW, Creemers DHM, Engels RCME, Granic I. The effect of the video game Mindlight on anxiety symptoms in children with an Autism Spectrum Disorder. BMC Psychiatry. 2015;15(1):1-9. doi:10.1186/s12888-015-0522-x

37. Wols A, Lichtwarck-Aschoff A, Schoneveld EA, Granic I. In-Game Play Behaviours during an Applied Video Game for Anxiety Prevention Predict Successful Intervention Outcomes. $J$ Psychopathol Behav Assess. 2018;40(4):655-668. doi:10.1007/s10862-018-9684-4

38. Ogino M, Kanoga S, Muto M, Mitsukura Y. Analysis of Prefrontal Single-Channel EEG Data for Portable Auditory ERP-Based Brain-Computer Interfaces. Front Hum Neurosci. 2019;13(July):1-14. 
doi:10.3389/fnhum.2019.00250

39. Lau-Zhu A, Lau MPH, McLoughlin G. Mobile EEG in research on neurodevelopmental disorders: Opportunities and challenges. Dev Cogn Neurosci. 2019;36(October 2018):100635. doi:10.1016/j.den.2019.100635

40. Swanson JM, Kramer HC, Hinshaw SP, et al. Clinical Relevance of the Primary Findings of the MTA: Success Rates Based on Severity of ADHD and ODD Symptoms at the End of Treatment. J Am Acad Child Adolesc Psychiatry. 2001;40(2):168-179. doi:10.1097/00004583-200102000-00011

41. Conners CK. A teacher rating scale for use in drug studies with children. Am J Psychiatry. 1969;126:884-888.

42. Loney J, Milich R. Hyperactivity, inattention, and aggression in clinical practice. In: Wolraich M, Routh DK, eds. Advances in Developmental and Behavioral Pediatrics. 3rd ed. Greenwich, CT: JAI; 1982:113-147.

43. Gau SS, Shang C, Liu S, et al. Psychometric properties of the Chinese version of the Swanson, Nolan, and Pelham, version IV scale - parent form. Int J Methods Psychiatr Res. 2008;17:35-44.

44. Waschbusch DA, Willoughby MT. Parent and teacher ratings on the IOWA Conners Rating Scale. $J$ Psychopathol Behav Assess. 2008;30(3):180-192. doi:10.1007/s10862-007-9064-y

45. Valderrama JT, De La Torre A, Van Dun B. An automatic algorithm for blink-artifact suppression based on iterative template matching: Application to single channel recording of cortical auditory evoked potentials. $J$ Neural Eng. 2018;15(1):016008, 15p. doi:10.1088/1741-2552/aa8d95

46. Parameshwaran D, Thiagarajan TC. Characterizing peaks in the EEG power spectrum. Biomed Phys Eng Express. 2019;5(4). doi:10.1088/2057-1976/ab29d0

47. Laufs H, Krakow K, Sterzer†, P. Eger† E, Beyerle† A, Salek-Haddad A, Kleinschmidt A. Electroencephalographic signatures of attentional and cognitive default modes in spontaneous brain activity fluctuations at rest. PNAS. 2003;100(19):11053-11058. doi:10.1084/jem.65.5.661

48. Kropotov JD. Quantitative EEG, Event-Related Potentials and Neurotherapy. 1st ed. San Diego: Elsevier; 2009.

49. Jensen O, Mazaheri A. Shaping functional architecture by oscillatory alpha activity: gating by inhibition. Front Hum Neurosci. 2010;4(November):186. doi:10.3389/fnhum.2010.00186

50. Klimesch W. Alpha-band oscillations, attention, and controlled access to stored information. Trends Cogn Sci. 2012;16(12):606-617. doi:10.1016/j.tics.2012.10.007

51. Bazanova OM, Vernon D. Interpreting EEG alpha activity. Neurosci Biobehav Rev. 2014;44:94-110. doi:10.1016/j.neubiorev.2013.05.007

52. Thut G, Nietzel A, Brandt S, Pascual-Leone A. Alpha Band Electroencephalographic Activity over 
Occipital Cortex Indexes Visuospatial Attention Bias and Predicts Visual Target Detection. J Neurosci. 2006;26(37):9494-9502. doi:10.1523/jneurosci.0875-06.2006

53. Bonnefond $\mathrm{M}$, Jensen $\mathrm{O}$. Alpha oscillations serve to protect working memory maintenance against anticipated distracters. Curr Biol. 2012;22(20):1969-1974. doi:10.1016/j.cub.2012.08.029

54. Meeuwissen EB, Takashima A, Fernández G, Jensen O. Increase in posterior alpha activity during rehearsal predicts successful long-term memory formation of word sequences. Hum Brain Mapp. 2011;32(12):2045-2053. doi:10.1002/hbm.21167

55. Sackett D, Haynes R, Guyatt G, Tugwell P. Clinical Epidemiology: A Basic Science for Clinical Medicine. 2nd ed. New York: Little Brown and co.; 1991.

56. Gasser T, Schuller JC, Gasser US. Correction of muscle artefacts in the EEG power spectrum. Clin Neurophysiol. 2005;116(9):2044-2050.

57. Barlow JS. MG artifact minimization during clinical EEG recordings by special analog filtering. Electroencephalogr Clin Neurophysiol. 1984;58(2):161-174.

58. Newson JJ, Thiagarajan TC. EEG Frequency Bands in Psychiatric Disorders: A Review of Resting State Studies. Front Hum Neurosci. 2019;12(January):1-24. doi:10.3389/fnhum.2018.00521

59. Kirkland AE, Holton KF. Measuring Treatment Response in Pharmacological and Lifestyle Interventions Using Electroencephalography in ADHD: A Review. Clin EEG Neurosci. 2019. doi:10.1177/1550059418817966

60. Glass G V, Peckham PD, Sanders JR. Consequences of Failure to Meet Assumptions Underlying the Fixed Effects Analyses of Variance and Covariance. Rev Educ Res. 1972;42(3):237-288. 
Table 1. Significance values for the main effects and interactions for the activation (EO-EC) and attentional activation (FO-EO) analyses.

\begin{tabular}{lcccccccccc}
\hline Condition & Band & \multicolumn{3}{c}{ Age main effect } & \multicolumn{3}{c}{ Condition main effect } & \multicolumn{2}{c}{ Age x Condition interaction } \\
\hline & & $\mathrm{F}$ & $\mathrm{p}$ & $\eta_{\mathrm{p}}^{2}$ & $\mathrm{~F}$ & $\mathrm{p}$ & $\eta_{\mathrm{p}}^{2}$ & $\mathrm{~F}$ & $\mathrm{p}$ & $\eta_{\mathrm{p}}^{2}$ \\
\hline EO-EC & Delta & 2.962 & .054 & 0.032 & 59.231 & .001 & 0.250 & 4.098 & .018 & 0.044 \\
& Theta & 6.535 & .002 & 0.067 & 47.768 & .001 & 0.212 & 3.236 & .042 & 0.035 \\
& Alpha & 3.681 & .027 & 0.040 & 121.259 & .001 & 0.405 & 5.588 & .004 & 0.059 \\
& Beta & 3.390 & .036 & 0.037 & 47.010 & .001 & 0.209 & & & \\
\multirow{5}{*}{ FO-EO } & Delta & & & & & & & & & \\
& Theta & & & & & & & & & \\
& Alpha & 3.043 & .050 & 0.033 & & & & & & \\
& Beta & & & & 7.531 & .007 & 0.040 & & & \\
\hline
\end{tabular}


Table 2. Percentage of participants that showed change in the expected direction for the significant Condition effects, for each age group separately.

\begin{tabular}{llcccc}
\hline Condition & Band & $7-8$ years & $9-10$ years & $11-12$ years & Mean \\
\hline EO-EC & Delta & 87.3 & 81.4 & 75.0 & 81.2 \\
& Theta & 85.7 & 81.4 & 73.8 & 80.3 \\
& Alpha & 73.5 & 87.1 & 95.2 & 85.3 \\
FO-EO & Beta & 77.3 & 79.1 & 72.2 & 76.2 \\
& Beta & 60.9 & 67.2 & 46.2 & 58.1 \\
\hline
\end{tabular}




\section{Delta}

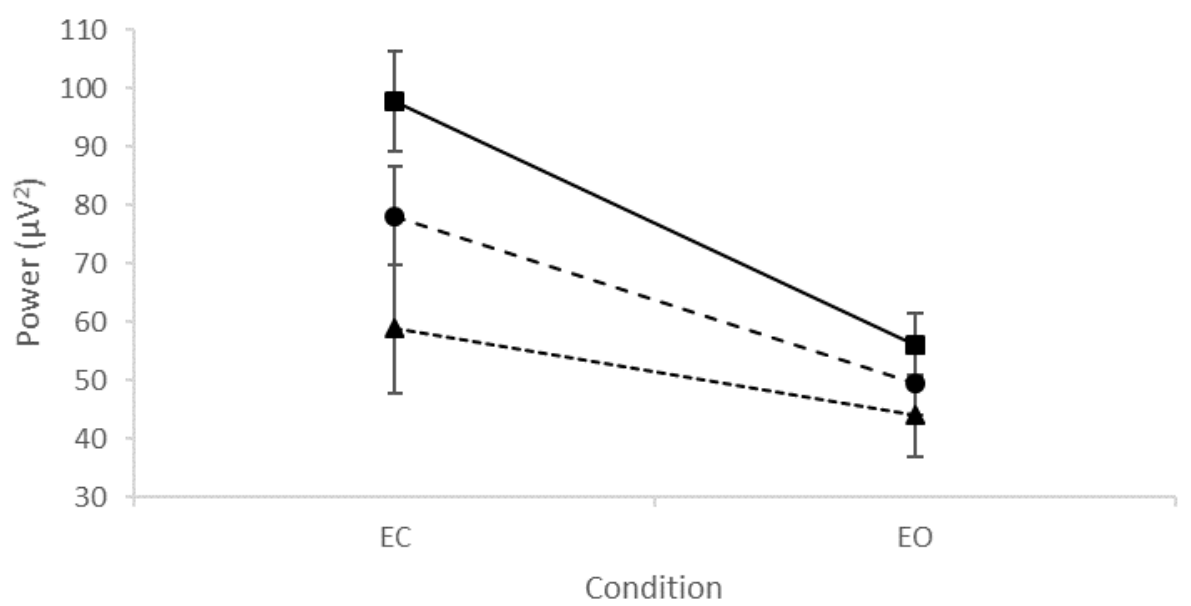

Theta
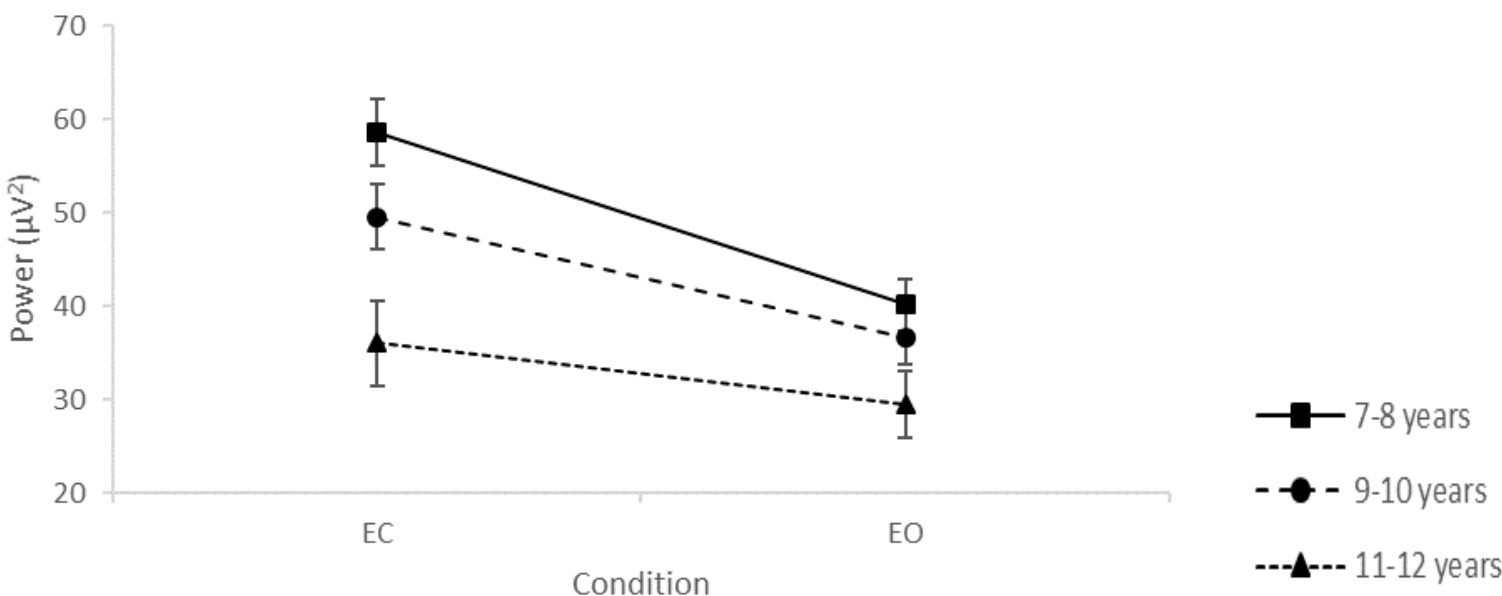

Alpha

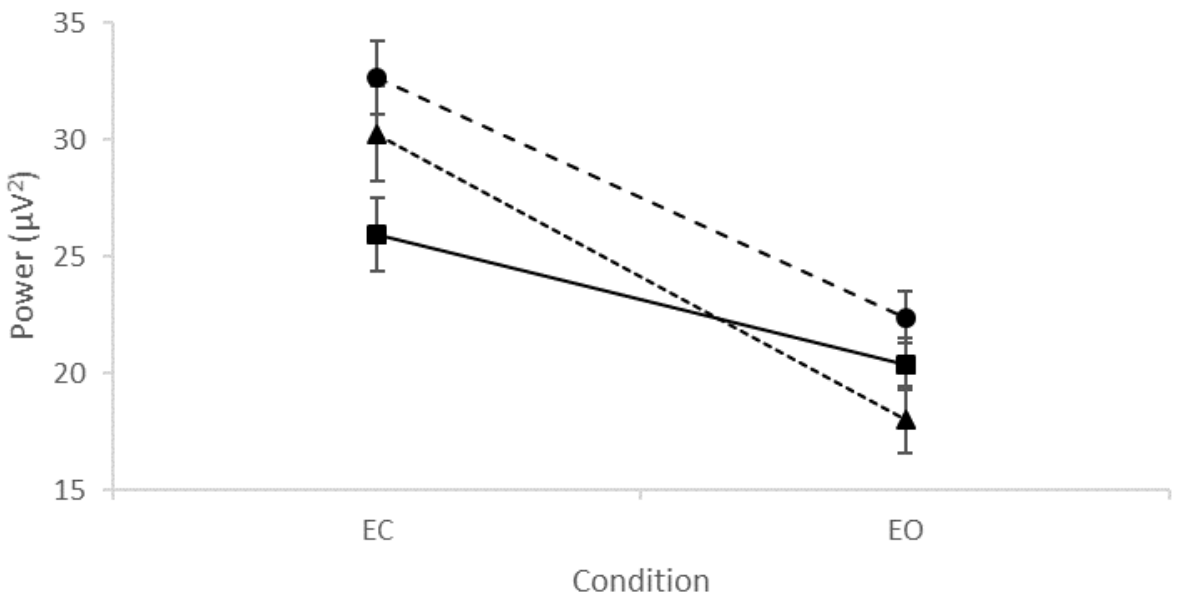

Figure 1. The Condition by Age interactions for frontal delta, theta, and alpha power. Error bars show standard error. EC, eyes-closed. EO, eyes-open. 


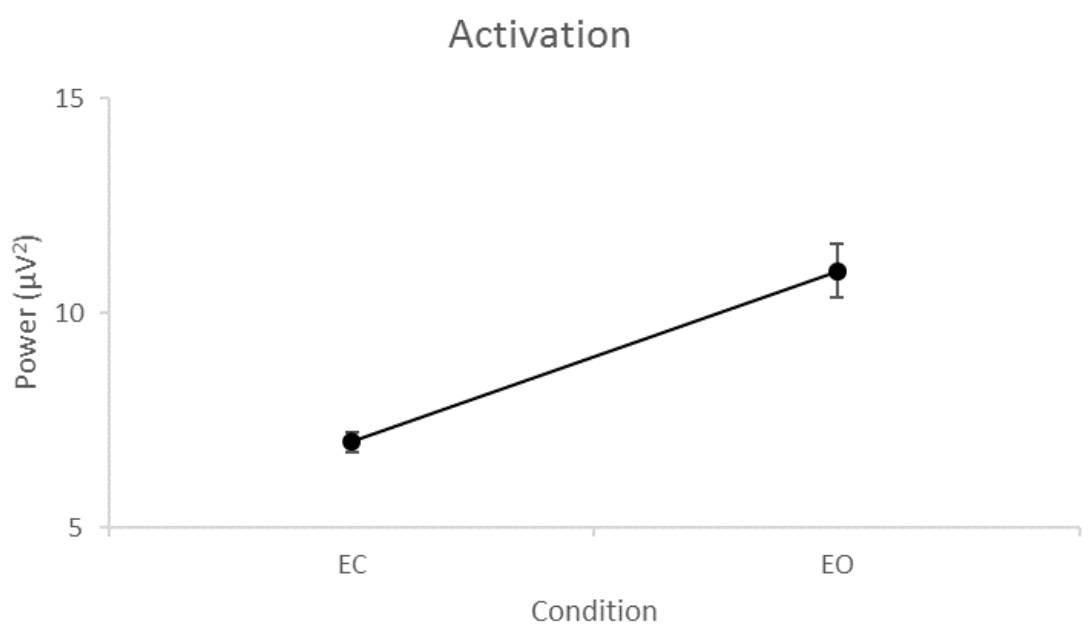

Attentional activation

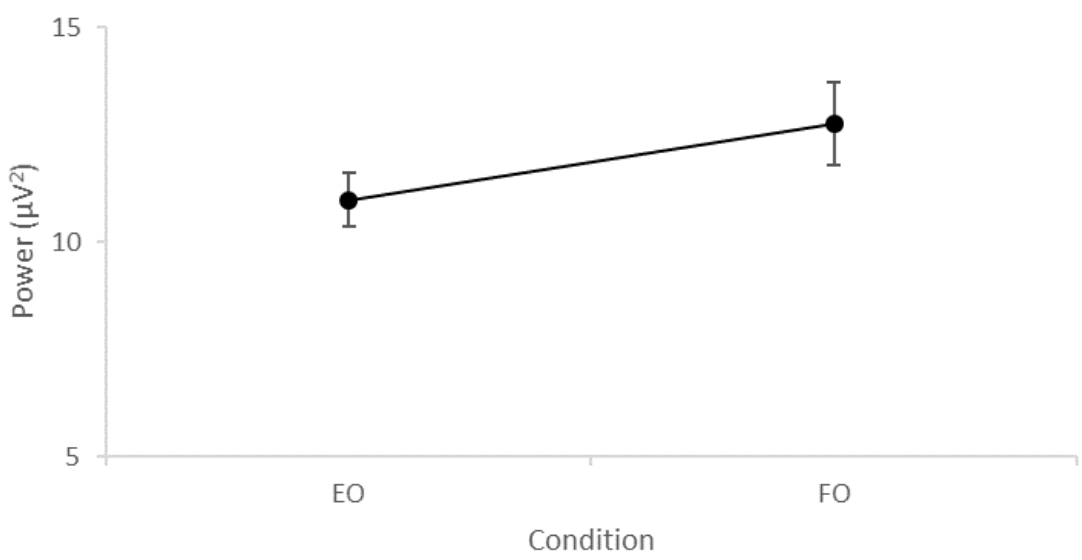

Figure 2. The Condition main effects for frontal beta activation and frontal beta attentional activation. Error bars show standard error. The Activation comparison involved quantification of the full $120 \mathrm{~s}$ trace for the EC and $\mathrm{EO}$ conditions. The Attentional activation comparison involved quantification of a randomly chosen $60 \mathrm{~s}$ trace from the EO condition and the $60 \mathrm{~s}$ trace of the FO condition. EC, eyes-closed. EO, eyes-open. 

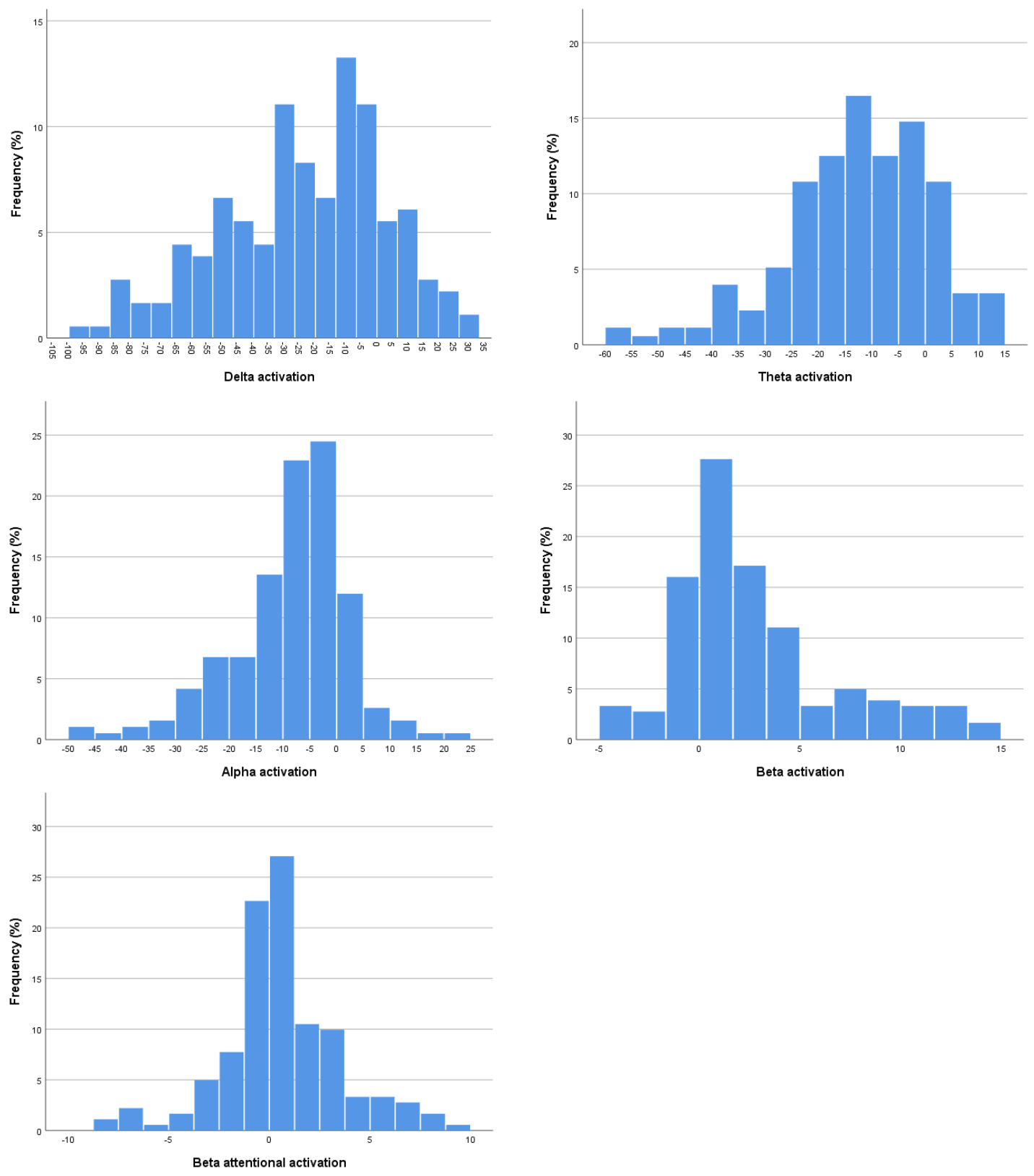

Figure 3. Histograms of the EO-EC (top 4 panels) and FO-EO (lower panel) difference scores (in $\mu \mathrm{V}^{2}$ ). Values below zero indicate an effect in the direction of the Condition effects for delta, theta, and alpha activation. Values above zero indicate an effect in the direction of the Condition effects for beta activation and beta attentional activation. 